\title{
Effective field theory with two Higgs doublets
}

\author{
Andreas Crivellin, ${ }^{a}$ Margherita Ghezzi ${ }^{a}$ and Massimiliano Procura ${ }^{b, 1}$ \\ ${ }^{a}$ Paul Scherrer Institut, \\ CH-5232 Villigen PSI, Switzerland \\ b Theoretical Physics Department, CERN, \\ Geneva, Switzerland \\ E-mail: andreas.crivellin@cern.ch, margherita.ghezzi@psi.ch, \\ massimiliano.procura@cern.ch
}

ABSTRACT: In this article we extend the effective field theory framework describing new physics effects to the case where the underlying low-energy theory is a Two-Higgs-Doublet model. We derive a complete set of independent operators up to dimension six assuming a $Z_{2}$-invariant $\mathrm{CP}$-conserving Higgs potential. The effects on Higgs and gauge boson masses, mixing angles in the Higgs sector as well as couplings to fermions and gauge bosons are computed. At variance with the case of a single Higgs doublet, we find that pair production of SM-like Higgses, arising through dimension-six operators, is not fixed by fermion-fermion-Higgs couplings and can therefore be sizable.

Keywords: Beyond Standard Model, Effective field theories

ArXIV EPRINT: 1608.00975

\footnotetext{
${ }^{1}$ On leave from the University of Vienna, Austria.
} 


\section{Contents}

1 Introduction 1

2 Notation and conventions 3

3 Gauge invariant operators 5

4 Physical basis $\quad 6$

4.1 Kinetic terms, Higgs and gauge boson masses 9

$\begin{array}{lll}4.2 & \text { Yukawa sector } & 12\end{array}$

5 Conclusions and outlook 13

\section{Introduction}

Adding a second $\mathrm{SU}(2)$ doublet scalar [1] represents one of the simplest possible extensions of the Standard Model (SM) of strong and electroweak (EW) interactions. Two-HiggsDoublet models (2HDMs) have been under extensive investigation for a long time (see for example ref. [2] for an introduction and ref. [3] for a more recent review article). There are several reasons for this interest. Firstly, these models have a rather small number of free parameters, which makes phenomenological analyses quite predictive. Additional motivation for $2 \mathrm{HDMs}$ is provided by axion models [4], where a global U(1) Peccei-Quinn symmetry is introduced to eliminate a CP-violating term in the QCD Lagrangian [5]. Such a symmetry is only possible in scenarios with at least two Higgs doublets. Furthermore, the amount of $\mathrm{CP}$ violation obtained through the introduction of a second Higgs doublet can be large enough to account for the baryon asymmetry of the universe [6]. 2HDMs can also explain the anomalies observed in tauonic $B$ decays [7-9]. Finally, strong motivation for studying $2 \mathrm{HDMs}$ is provided by the Minimal Supersymmetric Standard Model (MSSM) where supersymmetry enforces the introduction of a second Higgs doublet [10-12] due to the holomorphicity of the superpotential.

According to present collider bounds, the additional Higgs bosons contained in these models are still allowed to have masses around the EW scale $[13,14] .{ }^{1}$ Therefore, in an effective field theory (EFT) approach, it is natural to consider the additional Higgs doublet as a dynamical degree of freedom like the SM fields. In this article, we shall consider $2 \mathrm{HDMs}$ as EFTs valid up to a high-energy scale $\Lambda \gg M_{H}$ where additional dynamical degrees of freedom enter. The MSSM with heavy SUSY partners but light Higgs doublets is one

\footnotetext{
${ }^{1}$ An exception is the 2HDM of type II where the bound from $b \rightarrow s \gamma$ forces the charged Higgs mass (which only differs from the other Higgs masses by terms of order $v^{2}$ ) to be larger than $400 \mathrm{GeV}[15]$.
} 
example of such a theory, but also the $L_{\mu}-L_{\tau}$ model of refs. [16, 17] reduces to a $2 \mathrm{HDM}$ if the $Z^{\prime}$ and the $L_{\mu}-L_{\tau}$-breaking singlet are heavy.

In general, any theory of new physics (NP) super-seeding the 2HDM at higher energies must satisfy the following requirements (in close analogy to the SM case):

(i) Its gauge group contains the $\mathrm{SM}$ gauge group $\mathrm{SU}(3)_{C} \times \mathrm{SU}(2)_{L} \times \mathrm{U}(1)_{Y}$ as a subgroup.

(ii) It contains two Higgs doublets as dynamical degrees of freedom, either as fundamental or composite fields.

(iii) At low energies it reproduces the $2 \mathrm{HDM}$, barring the existence of weakly coupled light particles, like axions or sterile neutrinos (in which case the EFT should include in addition these particles as dynamical degrees of freedom).

In this approach, heavier NP particles are integrated out and their effects are parameterized in terms of Wilson coefficients of higher-dimension operators suppressed by inverse powers of $\Lambda$. In our $2 \mathrm{HDM}$ case we have

$$
\mathcal{L}_{2 H D M}=\mathcal{L}_{2 H D M}^{(4)}+\frac{1}{\Lambda} \sum_{k} C_{k}^{(5)} Q_{k}^{(5)}+\frac{1}{\Lambda^{2}} \sum_{k} C_{k}^{(6)} Q_{k}^{(6)}+\mathcal{O}\left(\frac{1}{\Lambda^{3}}\right)
$$

Here $\mathcal{L}_{2 H D M}^{(4)}$ is the standard renormalizable 2HDM Lagrangian (to be specified in the next section) which contains only dimension-two and dimension-four operators. $Q_{k}^{(5)}$ generalize the Weinberg operator [18] giving rise to neutrino masses and $Q_{k}^{(6)}$ denote the dimensionsix operators. $C_{k}^{(5)}$ and $C_{k}^{(6)}$ are their dimensionless Wilson coefficients. In this paper we neglect the effects of operators of dimension seven and higher, which are suppressed by at least three powers of $\Lambda$.

The EFT approach to parameterize NP effects through higher-dimension operators built with SM fields (SM-EFT) has been used for a long time [19-22]. More recently, a complete and minimal basis of dimension-six effective operators in the SM has been established [23] and the SM-EFT has received a lot of interest concerning its phenomenological applications (see for example [24-28]), mainly in the context of Higgs physics [29-37] but also flavor physics [38-47]. The possibility to extract constraints on the Wilson coefficients both from Higgs measurements at the LHC and from electroweak precision observables has been exploited [48-55] and an effort is currently made to perform the SM-EFT analysis at next-to-leading order in perturbation theory [56-67].

The purpose of this paper is to extend the SM-EFT approach to the case of two Higgs doublets. 2HDMs are considered here as low-energy theories and using our framework the effects of heavier NP particles can be clarified and studied in a systematic way. For example, effective operators in $2 \mathrm{HDM}$ save been recently considered to account for a diphoton excess in LHC data $[68,69]$ with the outcome that an explanation requires to extend the field content of pure 2HDMs [70-79]. ${ }^{2}$

\footnotetext{
${ }^{2}$ Prior to the LHC diphoton excess, effective operators for two-photon processes in 2 HDMs were already discussed in ref. [80].
} 


\begin{tabular}{|c|ccccc|c|}
\hline & \multicolumn{5}{|c|}{ fermions } & scalars \\
\hline field & $l_{L p}^{j}$ & $e_{R p}$ & $q_{L p}^{\alpha j}$ & $u_{R p}^{\alpha}$ & $d_{R p}^{\alpha}$ & $\varphi_{1}^{j}, \varphi_{2}^{j}$ \\
\hline hypercharge $Y$ & $-\frac{1}{2}$ & -1 & $\frac{1}{6}$ & $\frac{2}{3}$ & $-\frac{1}{3}$ & $\frac{1}{2}$ \\
\hline
\end{tabular}

Table 1. The matter content of the 2HDM. $l(e)$ is the lepton doublet (singlet), $u$ and $d$ the righthanded up and down quark singlets and $q$ the quark doublet. Here, $j=1,2, \alpha=1,2,3$, and $p=$ $1,2,3$ stand for isospin, color and generation indices, respectively.

This article is organized as follows. In section 2 we will introduce our notation and conventions. The complete list of operators up to dimension six before spontaneous EW symmetry breaking will be given in section 3 . We will then discuss EW symmetry breaking and the definition of the physical basis with diagonal mass matrices in section 4 . In section 5 we will conclude and point out an interesting phenomenological application of our formalism.

\section{Notation and conventions}

In this section we establish our notation and conventions following ref. [23] and ref. [3]. The renormalizable 2HDM Lagrangian before spontaneous EW symmetry breaking, reads

$$
\begin{aligned}
\mathcal{L}_{2 H D M}^{(4)}= & -\frac{1}{4} G_{\mu \nu}^{A} G^{A \mu \nu}-\frac{1}{4} W_{\mu \nu}^{I} W^{I \mu \nu}-\frac{1}{4} B_{\mu \nu} B^{\mu \nu} \\
& +\left(D_{\mu} \varphi_{1}\right)^{\dagger}\left(D^{\mu} \varphi_{1}\right)+\left(D_{\mu} \varphi_{2}\right)^{\dagger}\left(D^{\mu} \varphi_{2}\right) \\
& -V\left(\varphi_{1}, \varphi_{2}\right)+i(\bar{l} \not D l+\bar{q} \not D q+\bar{u} \not D u+\bar{d} \not D d)+\mathcal{L}_{Y},
\end{aligned}
$$

where $\varphi_{1}$ and $\varphi_{2}$ are the two Higgs doublets, $A=1 \ldots 8(I=1 \ldots 3)$ labels the $\mathrm{SU}(3)$ $(\mathrm{SU}(2))$ gauge bosons while $B_{\mu \nu}$ is the hypercharge field strength tensor. Note that we omitted the QCD $\theta$-term here. The fermion fields and their charges and representations are shown in table 1 . For the sake of simplicity, chirality indices are suppressed in the following. The conventions for covariant derivatives are fixed e.g. by

$$
\left(D_{\mu} q\right)_{\alpha j}=\left(\partial_{\mu}+i g_{s} T_{\alpha \beta}^{A} G_{\mu}^{A}+i g S_{j k}^{I} W_{\mu}^{I}+i g^{\prime} Y_{q} B_{\mu}\right) q^{\beta k} .
$$

with $T^{A}=\frac{1}{2} \lambda^{A}$ and $S^{I}=\frac{1}{2} \tau^{I}$ denoting the SU(3) and SU(2) generators, and $\lambda^{A}\left(\tau^{I}\right)$ the Gell-Mann (Pauli) matrices. It is useful to define the following Hermitian derivative terms:

$$
\varphi^{\dagger} i \stackrel{\leftrightarrow}{D}_{\mu} \varphi \equiv i \varphi^{\dagger} D_{\mu} \varphi-i\left(D_{\mu} \varphi\right)^{\dagger} \varphi \quad \text { and } \quad \varphi^{\dagger} i \stackrel{\leftrightarrow}{D_{\mu}^{I}} \varphi \equiv i \varphi^{\dagger} \tau^{I} D_{\mu} \varphi-i\left(D_{\mu} \varphi\right)^{\dagger} \tau^{I} \varphi
$$

The gauge field strength tensors are given by

$$
\begin{aligned}
G_{\mu \nu}^{A} & =\partial_{\mu} G_{\nu}^{A}-\partial_{\nu} G_{\mu}^{A}-g_{s} f^{A B C} G_{\mu}^{B} G_{\nu}^{C}, \\
W_{\mu \nu}^{I} & =\partial_{\mu} W_{\nu}^{I}-\partial_{\nu} W_{\mu}^{I}-g \epsilon^{I J K} W_{\mu}^{J} W_{\nu}^{K}, \\
B_{\mu \nu} & =\partial_{\mu} B_{\nu}-\partial_{\nu} B_{\mu}
\end{aligned}
$$

while $\tilde{X}_{\mu \nu}=\frac{1}{2} \epsilon_{\mu \nu \rho \sigma} X^{\rho \sigma}$, with $\epsilon_{0123}=+1$, denotes the dual tensor $\left(X=\left\{G^{A}, W^{I}, B\right\}\right)$. 
We consider a CP-conserving scalar potential [3]

$$
\begin{aligned}
V\left(\varphi_{1}, \varphi_{2}\right)= & m_{11}^{2} \varphi_{1}^{\dagger} \varphi_{1}+m_{22}^{2} \varphi_{2}^{\dagger} \varphi_{2}-m_{12}^{2}\left(\varphi_{1}^{\dagger} \varphi_{2}+\varphi_{2}^{\dagger} \varphi_{1}\right)+\frac{\lambda_{1}}{2}\left(\varphi_{1}^{\dagger} \varphi_{1}\right)^{2}+\frac{\lambda_{2}}{2}\left(\varphi_{2}^{\dagger} \varphi_{2}\right)^{2} \\
& +\lambda_{3} \varphi_{1}^{\dagger} \varphi_{1} \varphi_{2}^{\dagger} \varphi_{2}+\lambda_{4} \varphi_{1}^{\dagger} \varphi_{2} \varphi_{2}^{\dagger} \varphi_{1}+\frac{\lambda_{5}}{2}\left[\left(\varphi_{1}^{\dagger} \varphi_{2}\right)^{2}+\left(\varphi_{2}^{\dagger} \varphi_{1}\right)^{2}\right]
\end{aligned}
$$

with a $Z_{2}$ symmetry $^{3}$ (softly broken by a dimension-two term) preventing the existence of terms with odd powers of $\varphi_{1}$ and $\varphi_{2}$. Here all parameters are assumed to be real. ${ }^{4}$ In order to study fluctuations around the VEVs that minimize the potential, ${ }^{5}$ the two complex scalar fields are parameterized as

$$
\varphi_{a}=\left(\begin{array}{c}
\phi_{a}^{+} \\
\left(v_{a}+\rho_{a}+i \eta_{a}\right) / \sqrt{2}
\end{array}\right), \quad a=1,2 .
$$

The Lagrangian for the mass terms of the CP-odd $\left(\eta_{a}\right)$, CP-even $\left(\rho_{a}\right)$ and charged $\left(\phi_{a}^{+}\right)$ Higgses is

$$
L_{M_{H}}^{(4)}=\frac{1}{2}\left(\begin{array}{c}
\eta_{1} \\
\eta_{2}
\end{array}\right)^{T} m_{\eta}^{2}\left(\begin{array}{c}
\eta_{1} \\
\eta_{2}
\end{array}\right)+\left(\begin{array}{c}
\phi_{1}^{-} \\
\phi_{2}^{-}
\end{array}\right)^{T} m_{\phi^{ \pm}}^{2}\left(\begin{array}{c}
\phi_{1}^{+} \\
\phi_{2}^{+}
\end{array}\right)+\frac{1}{2}\left(\begin{array}{c}
\rho_{1} \\
\rho_{2}
\end{array}\right)^{T} m_{\rho}^{2}\left(\begin{array}{c}
\rho_{1} \\
\rho_{2}
\end{array}\right)
$$

with

$$
\begin{aligned}
& m_{\eta}^{2}=\left(v_{1} v_{2} \lambda_{5}-m_{12}^{2}\right)\left(\begin{array}{cc}
-\frac{v_{2}}{v_{1}} & 1 \\
1 & -\frac{v_{1}}{v_{2}}
\end{array}\right) \\
& m_{\rho}^{2}=\left(\begin{array}{cc}
\lambda_{1} v_{1}^{2}+m_{12}^{2} \frac{v_{2}}{v_{1}} & v_{1} v_{2}\left(\lambda_{3}+\lambda_{4}+\lambda_{5}\right)-m_{12}^{2} \\
v_{1} v_{2}\left(\lambda_{3}+\lambda_{4}+\lambda_{5}\right)-m_{12}^{2} & \lambda_{2} v_{2}^{2}+m_{12}^{2} \frac{v_{1}}{v_{2}}
\end{array}\right) \\
& m_{\phi^{ \pm}}^{2}=\left[\frac{v_{1} v_{2}}{2}\left(\lambda_{4}+\lambda_{5}\right)-m_{12}^{2}\right]\left(\begin{array}{cc}
-\frac{v_{2}}{v_{1}} & 1 \\
1 & -\frac{v_{1}}{v_{2}}
\end{array}\right)
\end{aligned}
$$

where we eliminated $m_{11}^{2}$ and $m_{22}^{2}$ by the minimization conditions. The charged and CPodd mass matrices have both one vanishing eigenvalue, which corresponds to the Goldstone bosons giving masses to the $W$ and the $Z$, and a non-zero eigenvalue named $m_{H^{ \pm}}^{2}$ and $m_{A}^{2}$, respectively. Both matrices are diagonalized by the same angle $\beta$ defined as

$$
\tan \beta \equiv v_{2} / v_{1} .
$$

Another independent rotation angle, called $\alpha$, enters the definition of the CP-even mass eigenstates $h$ and $H$, with eigenvalues $m_{h}$ and $m_{H}$ respectively:

$$
\begin{gathered}
h=\rho_{1} \sin \alpha-\rho_{2} \cos \alpha, \\
H=-\rho_{1} \cos \alpha-\rho_{2} \sin \alpha
\end{gathered}
$$

\footnotetext{
${ }^{3}$ Note that assigning Peccei-Quinn charges to Higgs doublets and fermions has the same effect on the potential and the Yukawa couplings as imposing a $Z_{2}$ symmetry.

${ }^{4}$ For an analysis of the conditions for a CP-conserving Higgs sector we refer to [81].

${ }^{5}$ Since no CP violation is involved, both $v_{1}$ and $v_{2}$ can be taken to be real.
} 


\begin{tabular}{|c|c|c|c|}
\hline model & $u_{R}$ & $d_{R}$ & $e_{R}$ \\
\hline Type I & $\varphi_{2}$ & $\varphi_{2}$ & $\varphi_{2}$ \\
Type II & $\varphi_{2}$ & $\varphi_{1}$ & $\varphi_{1}$ \\
Lepton - specific & $\varphi_{2}$ & $\varphi_{2}$ & $\varphi_{1}$ \\
Flipped & $\varphi_{2}$ & $\varphi_{1}$ & $\varphi_{2}$ \\
\hline
\end{tabular}

Table 2. Couplings of right-handed fermion singlets to Higgs doublets in $2 \mathrm{HDMs}$ with natural flavor conservation. These couplings can be enforced by an appropriate assignment of $Z_{2}$ (or Peccei-Quinn) charges to the scalar doublets and right-handed fermions.

where usually the lighter one is identified with the SM Higgs, with mass $m_{h} \approx 125 \mathrm{GeV}$.

Let us finally turn to the Yukawa part of the Lagrangian in eq. (2.1):

$$
\mathcal{L}_{Y}=-Y_{1}^{e} \bar{l} \varphi_{1} e-Y_{2}^{e} \bar{l} \varphi_{2} e-Y_{1}^{d} \bar{q} \varphi_{1} d-Y_{2}^{d} \bar{q} \varphi_{2} d-Y_{1}^{u} \bar{q} \widetilde{\varphi}_{1} u-Y_{2}^{u} \bar{q} \widetilde{\varphi}_{2} u+h . c .
$$

Here we suppressed fermion flavor indices and defined $\widetilde{\varphi}^{j}=\epsilon_{j k}\left(\varphi^{k}\right)^{\star}$, using the totally antisymmetric $\epsilon_{j k}$ with $\epsilon_{12}=+1$. The Yukawa couplings $Y_{1,2}^{f}$ are understood to be $3 \times 3$ matrices in flavor space. If $Y_{1}^{f}$ and $Y_{2}^{f}$ are simultaneously non-zero, in general flavor changing neutral currents arise [82-84]. However, there are four 2HDMs with natural flavor conservation (see table 2) where only one of these couplings is present. As for CPconservation in the potential, this can be achieved by an appropriate $Z_{2}$ charge assignment to right-handed fermions. When we discuss the extension to dimension six, we will assume that the terms are made $Z_{2}$-invariant in the same way.

\section{Gauge invariant operators}

In this section we list the independent gauge invariant operators up to dimension six in the 2HDM-EFT. They are defined before the EW symmetry breaking takes place, meaning that they are given in the interaction basis, as the mass basis is not yet defined. After EW symmetry breaking, the fermions acquire masses and also the Higgs mass matrices receive additional contributions compared to the $2 \mathrm{HDM}$ with dimension-four operators only.

At dimension five the generalization of the Weinberg operator reads

$$
Q_{\nu \nu}^{11}=\left(\widetilde{\varphi}_{1}^{\dagger} l_{p}\right)^{T} C\left(\widetilde{\varphi}_{1}^{\dagger} l_{r}\right), \quad Q_{\nu \nu}^{22}=\left(\widetilde{\varphi}_{2}^{\dagger} l_{p}\right)^{T} C\left(\widetilde{\varphi}_{2}^{\dagger} l_{r}\right)
$$

where $C$ denotes the charge conjugation matrix.

The procedure we follow to obtain a complete set of independent operators at dimension six is the same as the one applied and thoroughly described in ref. [23] for SM-EFT. Obviously, the operators involving no Higgs doublets do not change compared to SM-EFT, and for them we refer the reader to ref. [23]. Using classical equations of motion, neglecting total derivatives, and imposing the constraint of vanishing total hypercharge, we derived a set of independent operators, which we classify like in the case of the SM-EFT as follows: 


\begin{tabular}{|c|}
\hline$\varphi^{6}$ \\
\hline$Q_{\varphi}^{111}=\left(\varphi_{1}^{\dagger} \varphi_{1}\right)^{3}$ \\
$Q_{\varphi}^{112}=\left(\varphi_{1}^{\dagger} \varphi_{1}\right)^{2}\left(\varphi_{2}^{\dagger} \varphi_{2}\right)$ \\
$Q_{\varphi}^{122}=\left(\varphi_{1}^{\dagger} \varphi_{1}\right)\left(\varphi_{2}^{\dagger} \varphi_{2}\right)^{2}$ \\
$Q_{\varphi}^{222}=\left(\varphi_{2}^{\dagger} \varphi_{2}\right)^{3}$ \\
$Q_{\varphi}^{(1221) 1}=\left(\varphi_{1}^{\dagger} \varphi_{2}\right)\left(\varphi_{2}^{\dagger} \varphi_{1}\right)\left(\varphi_{1}^{\dagger} \varphi_{1}\right)$ \\
$Q_{\varphi}^{(1221) 2}=\left(\varphi_{1}^{\dagger} \varphi_{2}\right)\left(\varphi_{2}^{\dagger} \varphi_{1}\right)\left(\varphi_{2}^{\dagger} \varphi_{2}\right)$ \\
$Q_{\varphi}^{(1212) 1}=\left(\varphi_{1}^{\dagger} \varphi_{2}\right)^{2}\left(\varphi_{1}^{\dagger} \varphi_{1}\right)+$ h.c. \\
$Q_{\varphi}^{(1212) 2}=\left(\varphi_{1}^{\dagger} \varphi_{2}\right)^{2}\left(\varphi_{2}^{\dagger} \varphi_{2}\right)+$ h.c. \\
\hline
\end{tabular}

Table 3. Operators in the 2HDM-EFT containing six Higgs doublets.

- $\varphi^{6}$ : operators with Higgs doublets only (table 3 ), which modify the Higgs potential. We assumed that these operators respect the $Z_{2}$ symmetry present at dimension four.

- $\varphi^{4} D^{2}$ : operators with four Higgs doublets and two derivatives (table 4), which modify the kinetic terms of the Higgs fields, the Higgs-gauge boson interactions and the $W$ and $Z$ masses.

- $\Psi^{2} \varphi X$ : operators with two fermion fields, one field strength tensor and one Higgs doublet (table 5), which give rise to dipole interactions after EW symmetry breaking.

- $\varphi^{2} X^{2}$ : operators with two Higgs doublets and two field strength tensors (table 6).

- $\Psi^{2} \varphi^{2} D$ : operators with two fermions fields, two Higgs doublets and one covariant derivative (table 7 ), which contribute to the fermion- $Z$ and fermion- $W$ couplings after EW symmetry breaking.

- $\Psi^{2} \varphi^{3}$ : operators containing two fermion fields and three Higgs doublets (table 8), which modify the relation between fermion masses and Higgs-fermion couplings.

As in the case of the dimension-four Lagrangian, we assume that the discrete $Z_{2}$ symmetry for operators involving Higgs and fermion fields is restored by an appropriate charge assignment to right-handed fermions. Concerning table 4, we stress that, as in the case of one Higgs doublet, operators with derivatives acting on two conjugated or two unconjugated fields are not independent.

\section{Physical basis}

In this section we discuss the modifications of the Higgs potential and the relation between the Yukawa couplings and the fermion masses induced by the dimension-six contributions. 


\begin{tabular}{|c|c|c|}
\hline \multicolumn{3}{|c|}{$\varphi^{4} D^{2}$} \\
\hline$\square$ & & $\varphi D$ \\
\hline$Q_{\square}^{1(1)}=\left(\varphi_{1}^{\dagger} \varphi_{1}\right) \square\left(\varphi_{1}^{\dagger} \varphi_{1}\right)$ & $Q_{\varphi D}^{(1) 11(1)}=\left[\left(D_{\mu} \varphi_{1}\right)^{\dagger} \varphi_{1}\right]\left[\varphi_{1}^{\dagger}\left(D^{\mu} \varphi_{1}\right)\right]$ & $Q_{\varphi D}^{(1) 21(2)}=\left[\left(D_{\mu} \varphi_{1}\right)^{\dagger} \varphi_{2}\right]\left[\varphi_{1}^{\dagger}\left(D^{\mu} \varphi_{2}\right)\right]+$ h.c. \\
\hline$Q_{\square}^{2(2)}=\left(\varphi_{2}^{\dagger} \varphi_{2}\right) \square\left(\varphi_{2}^{\dagger} \varphi_{2}\right)$ & $Q_{\varphi D}^{(2) 22(2)}=\left[\left(D_{\mu} \varphi_{2}\right)^{\dagger} \varphi_{2}\right]\left[\varphi_{2}^{\dagger}\left(D^{\mu} \varphi_{2}\right)\right]$ & $Q_{\varphi D}^{(1) 12(2)}=\left[\left(D_{\mu} \varphi_{1}\right)^{\dagger} \varphi_{1}\right]\left[\varphi_{2}^{\dagger}\left(D^{\mu} \varphi_{2}\right)\right]+$ h.c. \\
\hline$Q_{\square}^{1(2)}=\left(\varphi_{1}^{\dagger} \varphi_{1}\right) \square\left(\varphi_{2}^{\dagger} \varphi_{2}\right)$ & $Q_{\varphi D}^{(1) 22(1)}=\left[\left(D_{\mu} \varphi_{1}\right)^{\dagger} \varphi_{2}\right]\left[\varphi_{2}^{\dagger}\left(D^{\mu} \varphi_{1}\right)\right]$ & $Q_{\varphi D}^{12(12)}=\left[\varphi_{1}^{\dagger} \varphi_{2}\right]\left[\left(D_{\mu} \varphi_{1}\right)^{\dagger}\left(D^{\mu} \varphi_{2}\right)\right]+$ h.c. \\
\hline$Q_{\square}^{2(1)}=\left(\varphi_{2}^{\dagger} \varphi_{2}\right) \square\left(\varphi_{1}^{\dagger} \varphi_{1}\right)$ & $Q_{\varphi D}^{(2) 11(2)}=\left[\left(D_{\mu} \varphi_{2}\right)^{\dagger} \varphi_{1}\right]\left[\varphi_{1}^{\dagger}\left(D^{\mu} \varphi_{2}\right)\right]$ & $Q_{\varphi D}^{12(21)}=\left[\varphi_{1}^{\dagger} \varphi_{2}\right]\left[\left(D_{\mu} \varphi_{2}\right)^{\dagger}\left(D^{\mu} \varphi_{1}\right)\right]+$ h.c. \\
\hline
\end{tabular}

Table 4. Operators with four Higgs doublets and two derivatives.

\begin{tabular}{|c|c|c|}
\hline \multicolumn{3}{|c|}{$\Psi^{2} \varphi X$} \\
\hline \multicolumn{1}{|c|}{$W$} & \multicolumn{1}{|c|}{$B$} \\
\hline$Q_{d G}^{1}=\left(\bar{q}_{p} \sigma^{\mu \nu} T^{A} d_{r}\right) \varphi_{1} G_{\mu \nu}^{A}$ & $Q_{d W}^{1}=\left(\bar{q}_{p} \sigma^{\mu \nu} d_{r}\right) \tau^{I} \varphi_{1} W_{\mu \nu}^{I}$ & $Q_{d B}^{1}=\left(\bar{q}_{p} \sigma^{\mu \nu} d_{r}\right) \varphi_{1} B_{\mu \nu}$ \\
$Q_{d G}^{2}=\left(\bar{q}_{p} \sigma^{\mu \nu} T^{A} d_{r}\right) \varphi_{2} G_{\mu \nu}^{A}$ & $Q_{d W}^{2}=\left(\bar{q}_{p} \sigma^{\mu \nu} d_{r}\right) \tau^{I} \varphi_{2} W_{\mu \nu}^{I}$ & $Q_{d B}^{2}=\left(\bar{q}_{p} \sigma^{\mu \nu} d_{r}\right) \varphi_{2} B_{\mu \nu}$ \\
$Q_{u G}^{1}=\left(\bar{q}_{p} \sigma^{\mu \nu} T^{A} u_{r}\right) \widetilde{\varphi}_{1} G_{\mu \nu}^{A}$ & $Q_{u W}^{1}=\left(\bar{q}_{p} \sigma^{\mu \nu} u_{r}\right) \tau^{I} \widetilde{\varphi}_{1} W_{\mu \nu}^{I}$ & $Q_{u B}^{1}=\left(\bar{q}_{p} \sigma^{\mu \nu} u_{r}\right) \widetilde{\varphi}_{1} B_{\mu \nu}$ \\
$Q_{u G}^{2}=\left(\bar{q}_{p} \sigma^{\mu \nu} T^{A} u_{r}\right) \widetilde{\varphi}_{2} G_{\mu \nu}^{A}$ & $Q_{u W}^{2}=\left(\bar{q}_{p} \sigma^{\mu \nu} u_{r}\right) \tau^{I} \widetilde{\varphi}_{2} W_{\mu \nu}^{I}$ & $Q_{u B}^{2}=\left(\bar{q}_{p} \sigma^{\mu \nu} u_{r}\right) \widetilde{\varphi}_{2} B_{\mu \nu}$ \\
& $Q_{e W}^{1}=\left(\bar{l}_{p} \sigma^{\mu \nu} e_{r}\right) \tau^{I} \varphi_{1} W_{\mu \nu}^{I}$ & $Q_{e B}^{1}=\left(\bar{l}_{p} \sigma^{\mu \nu} e_{r}\right) \varphi_{1} B_{\mu \nu}$ \\
$Q_{e W}^{2}=\left(\bar{l}_{p} \sigma^{\mu \nu} e_{r}\right) \tau^{I} \varphi_{2} W_{\mu \nu}^{I}$ & $Q_{e B}^{2}=\left(\bar{l}_{p} \sigma^{\mu \nu} e_{r}\right) \varphi_{2} B_{\mu \nu}$ \\
\hline
\end{tabular}

Table 5. Operators containing two fermion fields, one Higgs doublet and a field strength tensor. Here $\sigma^{\mu \nu}=i\left[\gamma^{\mu}, \gamma^{\nu}\right] / 2$.

\begin{tabular}{|c|c|}
\hline \multicolumn{2}{|c|}{$\varphi^{2} X^{2}$} \\
\hline$G G, W W, B B$ & $W B$ \\
\hline$Q_{\varphi X}^{11}=\left(\varphi_{1}^{\dagger} \varphi_{1}\right) X_{\mu \nu} X^{\mu \nu}$ & $Q_{\varphi W B}^{11}=\left(\varphi_{1}^{\dagger} \tau^{I} \varphi_{1}\right) W_{\mu \nu}^{I} B^{\mu \nu}$ \\
$Q_{\varphi X}^{22}=\left(\varphi_{2}^{\dagger} \varphi_{2}\right) X_{\mu \nu} X^{\mu \nu}$ & $Q_{\varphi W B}^{22}=\left(\varphi_{2}^{\dagger} \tau^{I} \varphi_{2}\right) W_{\mu \nu}^{I} B^{\mu \nu}$ \\
$Q_{\varphi \widetilde{X}}^{11}=\left(\varphi_{1}^{\dagger} \varphi_{1}\right) \widetilde{X}_{\mu \nu} X^{\mu \nu}$ & $Q_{\varphi \widetilde{W} B}^{11}=\left(\varphi_{1}^{\dagger} \tau^{I} \varphi_{1}\right) \widetilde{W}_{\mu \nu}^{I} B^{\mu \nu}$ \\
$Q_{\varphi \widetilde{X}}^{22}=\left(\varphi_{2}^{\dagger} \varphi_{2}\right) \widetilde{X}_{\mu \nu} X^{\mu \nu}$ & $Q_{\varphi \widetilde{W} B}^{22}=\left(\varphi_{2}^{\dagger} \tau^{I} \varphi_{2}\right) \widetilde{W}_{\mu \nu}^{I} B^{\mu \nu}$ \\
\hline
\end{tabular}

Table 6. Operators with two scalar fields and two field strength tensors. $X$ denotes $G^{A}, W^{I}$ or $B$. 


\begin{tabular}{|c|c|}
\hline \multicolumn{2}{|c|}{$\Psi^{2} \varphi^{2} D$} \\
\hline (1) & (3) \\
\hline$Q_{\varphi u d}^{1}=i\left(\widetilde{\varphi}_{1}^{\dagger} i \stackrel{\leftrightarrow}{D}_{\mu} \varphi_{1}\right)\left(\bar{u}_{p} \gamma^{\mu} d_{r}\right)$ & \\
\hline$Q_{\varphi u d}^{2}=i\left(\widetilde{\varphi}_{2}^{\dagger} i \stackrel{\leftrightarrow}{D}_{\mu} \varphi_{2}\right)\left(\bar{u}_{p} \gamma^{\mu} d_{r}\right)$ & \\
\hline$Q_{\varphi l}^{(1) 1}=\left(\varphi_{1}^{\dagger} i \stackrel{\leftrightarrow}{D}_{\mu} \varphi_{1}\right)\left(\bar{l}_{p} \gamma^{\mu} l_{r}\right)$ & $Q_{\varphi l}^{(3) 1}=\left(\varphi_{1}^{\dagger} i \stackrel{\leftrightarrow}{D_{\mu}^{I}} \varphi_{1}\right)\left(\bar{l}_{p} \tau^{I} \gamma^{\mu} l_{r}\right)$ \\
\hline$Q_{\varphi l}^{(1) 2}=\left(\varphi_{2}^{\dagger} i \stackrel{\leftrightarrow}{D}_{\mu} \varphi_{2}\right)\left(\bar{l}_{p} \gamma^{\mu} l_{r}\right)$ & $Q_{\varphi l}^{(3) 2}=\left(\varphi_{2}^{\dagger} i \stackrel{\leftrightarrow}{D_{\mu}^{I}} \varphi_{2}\right)\left(\bar{l}_{p} \tau^{I} \gamma^{\mu} l_{r}\right)$ \\
\hline$Q_{\varphi e}^{1}=\left(\varphi_{1}^{\dagger} i \stackrel{\leftrightarrow}{D}_{\mu} \varphi_{1}\right)\left(\bar{e}_{p} \gamma^{\mu} e_{r}\right)$ & \\
\hline$Q_{\varphi e}^{2}=\left(\varphi_{2}^{\dagger} i \stackrel{\leftrightarrow}{D}_{\mu} \varphi_{2}\right)\left(\bar{e}_{p} \gamma^{\mu} e_{r}\right)$ & \\
\hline$Q_{\varphi q}^{(1) 1}=\left(\varphi_{1}^{\dagger} i \stackrel{\leftrightarrow}{D}_{\mu} \varphi_{1}\right)\left(\bar{q}_{p} \gamma^{\mu} q_{r}\right)$ & $Q_{\varphi q}^{(3) 1}=\left(\varphi_{1}^{\dagger} i \stackrel{\leftrightarrow}{D_{\mu}^{I}} \varphi_{1}\right)\left(\bar{q}_{p} \tau^{I} \gamma^{\mu} q_{r}\right)$ \\
\hline$Q_{\varphi q}^{(1) 2}=\left(\varphi_{2}^{\dagger} i \stackrel{\leftrightarrow}{D}_{\mu} \varphi_{2}\right)\left(\bar{q}_{p} \gamma^{\mu} q_{r}\right)$ & $Q_{\varphi q}^{(3) 2}=\left(\varphi_{2}^{\dagger} i \stackrel{\leftrightarrow}{D_{\mu}^{I}} \varphi_{2}\right)\left(\bar{q}_{p} \tau^{I} \gamma^{\mu} q_{r}\right)$ \\
\hline$Q_{\varphi u}^{1}=\left(\varphi_{1}^{\dagger} i \stackrel{\leftrightarrow}{D}_{\mu} \varphi_{1}\right)\left(\bar{u}_{p} \gamma^{\mu} u_{r}\right)$ & \\
\hline$Q_{\varphi u}^{2}=\left(\varphi_{2}^{\dagger} i \stackrel{\leftrightarrow}{D}_{\mu} \varphi_{2}\right)\left(\bar{u}_{p} \gamma^{\mu} u_{r}\right)$ & \\
\hline$Q_{\varphi d}^{1}=\left(\varphi_{1}^{\dagger} i \stackrel{\leftrightarrow}{D}_{\mu} \varphi_{1}\right)\left(\bar{d}_{p} \gamma^{\mu} d_{r}\right)$ & \\
\hline$Q_{\varphi d}^{2}=\left(\varphi_{2}^{\dagger} i \stackrel{\leftrightarrow}{D}_{\mu} \varphi_{2}\right)\left(\bar{d}_{p} \gamma^{\mu} d_{r}\right)$ & \\
\hline
\end{tabular}

Table 7. Operators in the 2HDM-EFT containing two fermions, two Higgs doublets and a covariant derivative. The superscripts (3), (1) label fermion bilinears transforming as an $\mathrm{SU}(2)$ triplet or singlet, respectively.

\begin{tabular}{|c|c|c|}
\hline \multicolumn{3}{|c|}{$\Psi^{2} \varphi^{3}$} \\
\hline$e$ & $d$ & $u$ \\
\hline$Q_{e \varphi}^{111}=\left(\bar{l}_{p} e_{r} \varphi_{1}\right)\left(\varphi_{1}^{\dagger} \varphi_{1}\right)$ & $Q_{d \varphi}^{111}=\left(\bar{q}_{p} d_{r} \varphi_{1}\right)\left(\varphi_{1}^{\dagger} \varphi_{1}\right)$ & $Q_{u \varphi}^{111}=\left(\bar{q}_{p} u_{r} \widetilde{\varphi}_{1}\right)\left(\varphi_{1}^{\dagger} \varphi_{1}\right)$ \\
$Q_{e \varphi}^{122}=\left(\bar{l}_{p} e_{r} \varphi_{1}\right)\left(\varphi_{2}^{\dagger} \varphi_{2}\right)$ & $Q_{d \varphi}^{122}=\left(\bar{q}_{p} d_{r} \varphi_{1}\right)\left(\varphi_{2}^{\dagger} \varphi_{2}\right)$ & $Q_{u \varphi}^{122}=\left(\bar{q}_{p} u_{r} \widetilde{\varphi}_{1}\right)\left(\varphi_{2}^{\dagger} \varphi_{2}\right)$ \\
$Q_{e \varphi}^{222}=\left(\bar{l}_{p} e_{r} \varphi_{2}\right)\left(\varphi_{2}^{\dagger} \varphi_{2}\right)$ & $Q_{d \varphi}^{222}=\left(\bar{q}_{p} d_{r} \varphi_{2}\right)\left(\varphi_{2}^{\dagger} \varphi_{2}\right)$ & $Q_{u \varphi}^{222}=\left(\bar{q}_{p} u_{r} \widetilde{\varphi}_{2}\right)\left(\varphi_{2}^{\dagger} \varphi_{2}\right)$ \\
$Q_{e \varphi}^{211}=\left(\bar{l}_{p} e_{r} \varphi_{2}\right)\left(\varphi_{1}^{\dagger} \varphi_{1}\right)$ & $Q_{d \varphi}^{211}=\left(\bar{q}_{p} d_{r} \varphi_{2}\right)\left(\varphi_{1}^{\dagger} \varphi_{1}\right)$ & $Q_{u \varphi}^{211}=\left(\bar{q}_{p} u_{r} \widetilde{\varphi}_{2}\right)\left(\varphi_{1}^{\dagger} \varphi_{1}\right)$ \\
\hline
\end{tabular}

Table 8. Operators in the 2HDM-EFT containing two fermion fields and three Higgs doublets.

The operators affecting Higgs kinetic terms and Higgs potential (and thus the Higgs mass matrices) are given in table 4 and table 3, respectively. The modified relations between fermion masses and Higgs-fermion couplings stem from the operators in table 8. The kinetic terms of the gauge boson fields receive contributions from the operators in table 6 . 


\subsection{Kinetic terms, Higgs and gauge boson masses}

The effect of the operators in table 4 on the kinetic terms of the Higgs fields amounts to

$$
\begin{aligned}
L_{H_{\mathrm{kin}}}^{(4)+(6)}= & \frac{1}{2}\left(\begin{array}{c}
\partial_{\mu} \rho_{1} \\
\partial_{\mu} \rho_{2}
\end{array}\right)^{T}\left(\begin{array}{cc}
1+\frac{2 \Delta_{\square}^{11}}{\Lambda^{2}}+\frac{\Delta_{\varphi D}^{11}}{2 \Lambda^{2}} & \frac{\Delta_{\square}^{12}}{\Lambda^{2}}+\frac{\Delta_{\varphi D}^{12}}{2 \Lambda^{2}} \\
\frac{\Delta_{\square}^{12}}{\Lambda^{2}}+\frac{\Delta_{\varphi D}^{12}}{2 \Lambda^{2}} & 1+\frac{2 \Delta_{\square}^{22}}{\Lambda^{2}}+\frac{\Delta_{\varphi D}^{22}}{2 \Lambda^{2}}
\end{array}\right)\left(\begin{array}{c}
\partial_{\mu} \rho_{1} \\
\partial_{\mu} \rho_{2}
\end{array}\right) \\
& +\frac{1}{2}\left(\begin{array}{c}
\partial_{\mu} \eta_{1} \\
\partial_{\mu} \eta_{2}
\end{array}\right)^{T}\left(\begin{array}{cc}
1+\frac{\Delta_{\varphi D}^{11}}{2 \Lambda^{2}} & \frac{\Delta_{\varphi D}^{12}}{2 \Lambda^{2}} \\
\frac{\Delta_{\varphi D}^{12}}{2 \Lambda^{2}} & 1+\frac{\Delta_{\varphi D}^{22}}{2 \Lambda^{2}}
\end{array}\right)\left(\begin{array}{c}
\partial_{\mu} \eta_{1} \\
\partial_{\mu} \eta_{2}
\end{array}\right) \\
& +\left(\begin{array}{c}
\partial_{\mu} \phi_{1}^{+} \\
\partial_{\mu} \phi_{2}^{+}
\end{array}\right)^{\dagger}\left(\begin{array}{cc}
1 & \frac{\Delta_{\varphi D}^{+}}{2 \Lambda^{2}} \\
\frac{\Delta_{\varphi D}^{+}}{2 \Lambda^{2}} & 1
\end{array}\right)\left(\begin{array}{c}
\partial_{\mu} \phi_{1}^{+} \\
\partial_{\mu} \phi_{2}^{+}
\end{array}\right)
\end{aligned}
$$

with

$$
\begin{aligned}
\Delta_{\square}^{11} & =-C_{\square}^{1(1)} v_{1}^{2} \\
\Delta_{\square}^{12} & =-v_{1} v_{2}\left(C_{\square}^{1(2)}+C_{\square}^{2(1)}\right) \\
\Delta_{\square}^{22} & =-C_{\square}^{2(2)} v_{2}^{2} \\
\Delta_{\varphi D}^{11} & =C_{\varphi D}^{(1) 11(1)} v_{1}^{2}+C_{\varphi D}^{(1) 22(1)} v_{2}^{2} \\
\Delta_{\varphi D}^{22} & =C_{\varphi D}^{(2) 11(2)} v_{1}^{2}+C_{\varphi D}^{(2) 22(2)} v_{2}^{2} \\
\Delta_{\varphi D}^{12} & =v_{1} v_{2}\left(C_{\varphi D}^{(1) 12(2)}+C_{\varphi D}^{(1) 21(2)}+C_{\varphi D}^{12(21)}+C_{\varphi D}^{12(12)}\right) \\
\Delta_{\varphi D}^{+} & =v_{1} v_{2}\left(C_{\varphi D}^{12(21)}+C_{\varphi D}^{12(12)}\right) .
\end{aligned}
$$

The kinetic terms are made canonical by the shifts

$$
\begin{aligned}
& \rho_{1} \rightarrow \rho_{1}\left(1-\frac{\Delta_{\varphi D}^{11}+4 \Delta_{\square}^{11}}{4 \Lambda^{2}}\right)-\left(\frac{\Delta_{\varphi D}^{12}+4 \Delta_{\square}^{12}}{4 \Lambda^{2}}\right) \rho_{2} \\
& \rho_{2} \rightarrow \rho_{2}\left(1-\frac{\Delta_{\varphi D}^{22}+4 \Delta_{\square}^{22}}{4 \Lambda^{2}}\right)-\frac{\Delta_{\varphi D}^{12}+4 \Delta_{\square}^{12}}{4 \Lambda^{2}} \rho_{1} \\
& \eta_{1} \rightarrow \eta_{1}\left(1-\frac{\Delta_{\varphi D}^{11}}{4 \Lambda^{2}}\right)-\frac{\Delta_{\varphi D}^{12}}{4 \Lambda^{2}} \eta_{2} \\
& \eta_{2} \rightarrow \eta_{2}\left(1-\frac{\Delta_{\varphi D}^{22}}{4 \Lambda^{2}}\right)-\frac{\Delta_{\varphi D}^{12}}{4 \Lambda^{2}} \eta_{1} \\
& \phi_{1}^{+} \rightarrow \phi_{1}^{+}-\frac{\Delta_{\varphi D}^{+}}{4 \Lambda^{2}} \phi_{2}^{+} \\
& \phi_{2}^{+} \rightarrow \phi_{2}^{+}-\frac{\Delta_{\varphi D}^{+}}{4 \Lambda^{2}} \phi_{1}^{+} .
\end{aligned}
$$


The operators with gauge and Higgs fields in table 6 lead to the shifts

$$
\begin{aligned}
W_{\mu}^{ \pm} & \rightarrow W_{\mu}^{ \pm}\left(1+\frac{\Delta_{W W}}{\Lambda^{2}}\right) \\
Z_{\mu} & \rightarrow Z_{\mu}\left(1+\frac{\Delta_{Z Z}}{\Lambda^{2}}\right) \\
A_{\mu} & \rightarrow A_{\mu}\left(1+\frac{\Delta_{A A}}{\Lambda^{2}}\right)+Z_{\mu} \frac{\Delta_{A Z}}{\Lambda^{2}} \\
G_{\mu}^{A} & \rightarrow G_{\mu}^{A}\left(1+\frac{\Delta_{G G}}{\Lambda^{2}}\right)
\end{aligned}
$$

with

$$
\begin{aligned}
\Delta_{G G} & =v_{1}^{2} C_{\varphi G}^{11}+v_{2}^{2} C_{\varphi G}^{22} \\
\Delta_{W W} & =v_{1}^{2} C_{\varphi W}^{11}+v_{2}^{2} C_{\varphi W}^{22} \\
\Delta_{Z Z} & =c_{w}^{2}\left(v_{1}^{2} C_{\varphi W}^{11}+v_{2}^{2} C_{\varphi W}^{22}\right)+s_{w}^{2}\left(v_{1}^{2} C_{\varphi B}^{11}+v_{2}^{2} C_{\varphi B}^{22}\right)+c_{w} s_{w}\left(v_{1}^{2} C_{\varphi W B}^{11}+v_{2}^{2} C_{\varphi W B}^{22}\right) \\
\Delta_{A Z} & =2 c_{w} s_{w}\left[v_{1}^{2}\left(C_{\varphi W}^{11}-C_{\varphi B}^{11}\right)+v_{2}^{2}\left(C_{\varphi W}^{22}-C_{\varphi B}^{22}\right)\right]+\left(s_{w}^{2}-c_{w}^{2}\right)\left(v_{1}^{2} C_{\varphi W B}^{11}+v_{2}^{2} C_{\varphi W B}^{22}\right) \\
\Delta_{A A} & =s_{w}^{2}\left(v_{1}^{2} C_{\varphi W}^{11}+v_{2}^{2} C_{\varphi W}^{22}\right)+c_{w}^{2}\left(v_{1}^{2} C_{\varphi B}^{11}+v_{2}^{2} C_{\varphi B}^{22}\right)-c_{w} s_{w}\left(v_{1}^{2} C_{\varphi W B}^{11}+v_{2}^{2} C_{\varphi W B}^{22}\right)
\end{aligned}
$$

where $c_{w}$ and $s_{w}$ denote cosine and sine of the weak mixing angle, respectively. These relations, together with the $\varphi D$ operators, affect the $W$ and $Z$ masses as

$$
\begin{aligned}
M_{Z}^{2} & =\frac{1}{4}\left(g^{2}+g^{\prime 2}\right)\left(v^{2}+\frac{4 v^{2} \Delta_{Z Z}+v_{1}^{2} \Delta_{\varphi D}^{11}+v_{2}^{2} \Delta_{\varphi D}^{22}+2 v_{1} v_{2} \Delta_{\varphi D}^{12}}{2 \Lambda^{2}}\right) \\
M_{W}^{2} & =\frac{1}{4} g^{2}\left(v^{2}+\frac{2 v^{2} \Delta_{W W}+v_{1} v_{2} \Delta_{\varphi D}^{+}}{\Lambda^{2}}\right)
\end{aligned}
$$

where $v^{2} \equiv v_{1}^{2}+v_{2}^{2}$.

The shift of the Higgs fields applied to the dimension-four potential and the $\varphi^{6}$ operators modify the Higgs mass terms in the Lagrangian. Choosing again to eliminate $m_{11}^{2}$ and $m_{22}^{2}$ via the minimization conditions, we obtained

$$
\begin{aligned}
L_{M_{H}}^{(4)+(6)}= & \frac{1}{2}\left(\begin{array}{c}
\eta_{1} \\
\eta_{2}
\end{array}\right)^{T}\left(m_{\eta}^{2}+\Delta m_{\eta}^{2}\right)\left(\begin{array}{c}
\eta_{1} \\
\eta_{2}
\end{array}\right)+\left(\begin{array}{c}
\phi_{1}^{-} \\
\phi_{2}^{-}
\end{array}\right)^{T}\left(m_{\phi^{ \pm}}^{2}+\Delta m_{\phi^{ \pm}}^{2}\right)\left(\begin{array}{c}
\phi_{1}^{+} \\
\phi_{2}^{+}
\end{array}\right) \\
& +\frac{1}{2}\left(\begin{array}{c}
\rho_{1} \\
\rho_{2}
\end{array}\right)^{T}\left(m_{\rho}^{2}+\Delta m_{\rho}^{2}\right)\left(\begin{array}{c}
\rho_{1} \\
\rho_{2}
\end{array}\right)
\end{aligned}
$$

with

$$
\begin{gathered}
\Delta m_{\eta}^{2}=\Delta m_{\varphi D \eta}^{2}+\Delta m_{\varphi^{6} \eta}^{2} \\
\Delta m_{\rho}^{2}=\Delta m_{\varphi D \rho}^{2}+\Delta m_{\varphi^{6} \rho}^{2} \\
\Delta m_{\phi^{ \pm}}^{2}=\Delta m_{\varphi D \phi^{ \pm}}^{2}+\Delta m_{\varphi^{6} \phi^{ \pm}}^{2}
\end{gathered}
$$


and

$$
\begin{aligned}
& \Delta m_{\varphi D \phi^{ \pm}}^{2}=\left[\left(\lambda_{4}+\lambda_{5}\right) v_{1} v_{2}-2 m_{12}^{2}\right] \frac{\Delta_{\varphi \mathrm{D}}^{+}}{4 \Lambda^{2}}\left(\begin{array}{cc}
-1 & \frac{v^{2}}{2 v_{1} v_{2}} \\
\frac{v^{2}}{2 v_{1} v_{2}} & -1
\end{array}\right) \\
& \left(\Delta m_{\varphi D \rho}^{2}\right)_{11}=\frac{\left(v_{1} \Delta_{\varphi \mathrm{D}}^{12}-v_{2} \Delta_{\varphi \mathrm{D}}^{11}\right) m_{12}^{2}-v_{1}^{2}\left[v_{1} \Delta_{\varphi \mathrm{D}}^{11} \lambda_{1}+v_{2} \Delta_{\varphi \mathrm{D}}^{12}\left(\lambda_{3}+\lambda_{4}+\lambda_{5}\right)\right]}{2 \Lambda^{2} v_{1}} \\
& \left(\Delta m_{\varphi D \rho}^{2}\right)_{12}=\frac{\left[-v^{2} \Delta_{\varphi \mathrm{D}}^{12}+v_{1} v_{2}\left(\Delta_{\varphi \mathrm{D}}^{22}+\Delta_{\varphi \mathrm{D}}^{11}\right)\right] m_{12}^{2}}{4 \Lambda^{2} v_{1} v_{2}} \\
& +\frac{v_{1} v_{2}\left(-\Delta_{\varphi \mathrm{D}}^{22}-\Delta_{\varphi \mathrm{D}}^{11}\right)\left(\lambda_{3}+\lambda_{4}+\lambda_{5}\right)-\left(v_{1}^{2} \lambda_{1}+v_{2}^{2} \lambda_{2}\right) \Delta_{\varphi \mathrm{D}}^{12}}{4 \Lambda^{2}} \\
& \left(\Delta m_{\varphi D \rho}^{2}\right)_{22}=\frac{\left(v_{2} \Delta_{\varphi \mathrm{D}}^{12}-v_{1} \Delta_{\varphi \mathrm{D}}^{22}\right) m_{12}^{2}-v_{2}^{2}\left[v_{2} \Delta_{\varphi \mathrm{D}}^{22} \lambda_{2}+v_{1} \Delta_{\varphi \mathrm{D}}^{12}\left(\lambda_{3}+\lambda_{4}+\lambda_{5}\right)\right]}{2 \Lambda^{2} v_{2}} \\
& \Delta m_{\varphi D \eta}^{2}=\frac{m_{12}^{2}-v_{1} v_{2} \lambda_{5}}{2 \Lambda^{2} v_{1} v_{2}}\left(\begin{array}{cc}
v_{1} v_{2} \Delta_{\varphi \mathrm{D}}^{12}-v_{2}^{2} \Delta_{\varphi \mathrm{D}}^{11} & \frac{v_{1} v_{2}\left(\Delta_{\varphi \mathrm{D}}^{22}+\Delta_{\varphi \mathrm{D}}^{11}\right)-v^{2} \Delta_{\varphi \mathrm{D}}^{12}}{2} \\
\frac{v_{1} v_{2}\left(\Delta_{\varphi \mathrm{D}}^{22}+\Delta_{\varphi \mathrm{D}}^{11}\right)-v^{2} \Delta_{\varphi \mathrm{D}}^{12}}{2} & v_{1} v_{2} \Delta_{\varphi \mathrm{D}}^{12}-v_{1}^{2} \Delta_{\varphi \mathrm{D}}^{22}
\end{array}\right)
\end{aligned}
$$

as well as

$$
\begin{gathered}
\Delta m_{\varphi^{6} \eta}^{2}=\Delta A\left(\begin{array}{cc}
-v_{2} / v_{1} & 1 \\
1 & -v_{1} / v_{2}
\end{array}\right) \\
\Delta m_{\varphi^{6} \phi^{ \pm}}^{2}=\Delta H^{ \pm}\left(\begin{array}{cc}
-v_{2} / v_{1} & 1 \\
1 & -v_{1} / v_{2}
\end{array}\right)
\end{gathered}
$$

with

$$
\begin{aligned}
\Delta H^{ \pm} & =\frac{v_{1} v_{2}}{2 \Lambda^{2}}\left[\left(C_{\varphi}^{(1212) 1}+\frac{1}{2} C_{\varphi}^{(1221) 1}\right) v_{1}^{2}+\left(C_{\varphi}^{(1212) 2}+\frac{1}{2} C_{\varphi}^{(1221) 2}\right) v_{2}^{2}\right] \\
\Delta A & =\frac{v_{1} v_{2}}{\Lambda^{2}}\left(C_{\varphi}^{(1212) 1} v_{1}^{2}+C_{\varphi}^{(1212) 2} v_{2}^{2}\right)
\end{aligned}
$$

and

$$
\begin{aligned}
& \left(\Delta m_{\varphi^{6} \rho}^{2}\right)_{11}=\frac{v_{1}^{2}}{\Lambda^{2}}\left(3 C_{\varphi}^{111} v_{1}^{2}+\left(C_{\varphi}^{112}+2 C_{\varphi}^{(1212) 1}+C_{\varphi}^{(1221) 1}\right) v_{2}^{2}\right) \\
& \left(\Delta m_{\varphi^{6} \rho}^{2}\right)_{12}=\frac{v_{1} v_{2}}{\Lambda^{2}}\left[\left(C_{\varphi}^{112}+2 C_{\varphi}^{(1212) 1}+C_{\varphi}^{(1221) 1}\right) v_{1}^{2}+\left(C_{\varphi}^{122}+2 C_{\varphi}^{(1212) 2}+C_{\varphi}^{(1221) 2}\right) v_{2}^{2}\right] \\
& \left(\Delta m_{\varphi^{6} \rho}^{2}\right)_{22}=\frac{v_{2}^{2}}{\Lambda^{2}}\left[\left(C_{\varphi}^{122}+2 C_{\varphi}^{(1212) 2}+C_{\varphi}^{(1221) 2}\right) v_{1}^{2}+3 C_{\varphi}^{222} v_{2}^{2}\right] .
\end{aligned}
$$

Interestingly, the mass matrices for the CP-odd and the charged Higgs bosons are not diagonalized anymore by the angle $\beta$ as in the case of the dimension-four potential. For this purpose, one needs two angles $\beta_{\eta}$ and $\beta_{\phi^{ \pm}}$, which do not satisfy $\tan \beta_{\eta, \phi^{ \pm}}=v_{1} / v_{2}$. 
This relation holds at dimension four but is broken by the $\varphi D$ operators. However, in the presence of $\square$ and $\varphi^{6}$ operators only, the CP-odd and the charged Higgs mass matrices would still be diagonalized by $\beta$ defined as $\tan \beta=v_{1} / v_{2}$. Also the angle $\alpha$ diagonalizing the CP-even mass matrix gets modified compared to the case of $2 \mathrm{HDM}$ without dimensionsix operators. However, this effect can be accounted for by an appropriate redefinition of $\alpha$, which is anyway a free parameter in the $2 \mathrm{HDM} .{ }^{6}$ The eigenvalues of the mass matrices also change, except those corresponding to the pseudo-Goldstone bosons, which are still zero at $\mathcal{O}\left(1 / \Lambda^{2}\right)$, as we checked. Therefore, the effects on the eigenvalues can be absorbed into the definitions of $m_{h}, m_{H}, m_{H^{ \pm}}$and $m_{A}$.

\subsection{Yukawa sector}

After EW symmetry breaking, the fermion mass matrices in the presence of the dimensionsix operators of table 8 are given by

$$
m^{f}=\frac{v_{1} Y_{1}^{f}}{\sqrt{2}}+\frac{v_{2} Y_{2}^{f}}{\sqrt{2}}+\frac{1}{2 \sqrt{2} \Lambda^{2}}\left(v_{1}^{3} C_{f \varphi}^{111}+v_{1} v_{2}^{2} C_{f \varphi}^{122}+v_{2}^{3} C_{f \varphi}^{222}+v_{1}^{2} v_{2} C_{f \varphi}^{211}\right)
$$

with $f=e, d, u$. Here $m^{f}, Y_{1,2}^{f}$ as well as the coefficients of the dimension-six operators are in general arbitrary $3 \times 3$ matrices in flavor space. However, since the eigenvalues of $m^{f}$ are the physical fermion masses, by working in the basis where $m^{f}$ is diagonal, the rotations that map onto this basis get implicitly absorbed in the definitions of $Y_{1,2}^{f}$ and $C_{f \varphi}$.

Depending on which version of the four $2 \mathrm{HDMs}$ with natural flavor conservation we are interested in (or which one is assumed to be the limiting case of the $2 \mathrm{HDM}$ of type $\mathrm{III}^{7}$ ), one can choose to eliminate either $Y_{1}^{f}$ or $Y_{2}^{f}$ from the Yukawa Lagrangian. Afterwards, it is straightforward to calculate the couplings of $\phi_{1,2}^{+}, \eta_{1,2}$ and $\rho_{1,2}$ to fermions.

Taking as an example the lepton Yukawa couplings and eliminating $Y_{1}^{e}$, we find

$$
\begin{aligned}
\mathcal{L}_{Y^{e}}^{(6)}= & \frac{i}{v_{1}}\left(m^{e}-\frac{v_{2}}{2 \sqrt{2}} \epsilon^{e}\right) \bar{l} P_{R} e \eta_{1}+\frac{i}{2 \sqrt{2}} \epsilon^{e} \bar{l} P_{R} e \eta_{2} \\
& +\frac{1}{v_{1}}\left(\sqrt{2} m^{e}-\frac{v_{2}}{2} \epsilon^{e}\right) \bar{\nu} P_{R} e \phi_{1}^{+} \\
& +\frac{1}{2} \epsilon^{e} \bar{\nu} P_{R} e \phi_{2}^{+}+\left[\frac{C_{e \varphi}^{111} v_{1}^{2}+C_{e \varphi}^{211} v_{1} v_{2}}{\sqrt{2} \Lambda^{2}}+\frac{1}{v_{1}}\left(m^{e}-\frac{v_{2} \epsilon^{e}}{2 \sqrt{2}}\right)\right] \bar{l} P_{R} e \rho_{1} \\
& +\left(\frac{C_{e \varphi}^{222} v_{2}^{2}+C_{e \varphi}^{122} v_{1} v_{2}}{\sqrt{2} \Lambda^{2}}+\frac{\epsilon^{e}}{2 \sqrt{2}}\right) \bar{l} P_{R} e \rho_{2}
\end{aligned}
$$

with

$$
\epsilon^{e}=2 Y_{2}^{e}+\frac{C_{e \varphi}^{211} v_{1}^{2}+C_{e \varphi}^{222} v_{2}^{2}}{\Lambda^{2}}
$$

\footnotetext{
${ }^{6}$ Nevertheless, one should keep in mind that in the limit of heavy Higgses $A^{0}, H^{0}, H^{ \pm}$, $\alpha$ has to vanish as $\tan \beta \rightarrow \infty$ modulo corrections of order $v^{2} / m_{H}^{2}$. When dimension-six operators are included, this is still the case but additional (even smaller) corrections of order $v^{2} / \Lambda^{2}$ are present.

${ }^{7}$ Due to stringent flavor constraints (see ref. [85] for a recent analysis), a 2HDM with generic Yukawa couplings (i.e. of type III) should only slightly differ from one of the four 2HDMs with natural flavor conservation.
} 
and $P_{R}$ denoting the right-handed projector. The outcome in the case that $Y_{2}^{e}$ is eliminated is obtained by simply interchanging $1 \leftrightarrow 2$. In order to express these couplings in terms of the physical Higgs fields, the rotations by (redefined) $\alpha, \beta_{\eta}$ and $\beta_{\phi^{ \pm}}$have to be applied. The analogous of eq. (4.14) for down-quarks has exactly the same structure. For up-type quarks all terms involving a charged or CP-odd Higgs switch sign and $\phi_{1,2}^{+}$get replaced by $\phi_{1,2}^{-}$.

We can now point out an interesting effect arising in the 2HDM-EFT related to Higgspair production [86-89]. In the SM-EFT there is only one operator giving rise to fermionfermion-Higgs-Higgs interactions and this is directly correlated to fermion-fermion-Higgs couplings $[60,90]$. Therefore, its effect in the four-particle interaction is limited due to the constraints on the fermion-fermion-Higgs couplings. In our 2HDM-EFT three terms affect the fermion-fermion-Higgs couplings while the part of the Lagrangian relevant for Higgs pair production is

$$
\frac{1}{2 \sqrt{2} \Lambda^{2}} \bar{f}\left(3 C_{f \varphi}^{111} v_{1} \rho_{1}^{2}+C_{f \varphi}^{122} v_{1} \rho_{2}^{2}+C_{f \varphi}^{122} v_{2} \rho_{1} \rho_{2}+C_{f \varphi}^{211} v_{1} \rho_{1} \rho_{2}+C_{f \varphi}^{211} v_{2} \rho_{1}^{2}+3 C_{f \varphi}^{222} v_{2} \rho_{2}^{2}\right) f .
$$

By comparing with eq. (4.14), it is clear that a cancellation between $C_{f \varphi}^{222}$ and $C_{f \varphi}^{122}$ could have quite different effects in fermion vertices with one or two Higgses. Therefore a suppressed modification to fermion-fermion-Higgs couplings accompanied by a sizable effect in pair production of SM-like Higgs bosons is possible in 2HDM-EFT.

\section{Conclusions and outlook}

In this article we extended the SM-EFT approach to NP to the case in which two Higgs doublets are dynamical degrees of freedom (2HDM-EFT). In this framework the effects of additional heavy particles (e.g. heavy SUSY partners in the MSSM) are parameterized in terms of higher-dimension operators and their Wilson coefficients. Our analysis enables systematic studies of the role played by additional degrees of freedom beyond the 2HDM field content. We derived a complete set of independent gauge-invariant dimension-six operators with two Higgs doublets of the same hypercharge under the assumption of a $Z_{2}$ symmetry involving the Higgs field and the right-handed fermions. These operators modify the Higgs potential and the relation between the fermion masses and Higgs-fermion-fermion couplings. We performed the transition to the physical basis by re-diagonalizing the Higgs, gauge boson and fermion kinetic terms and mass matrices. We showed that the CP-odd and charged Higgs mass matrices are in general not diagonalized by one angle $\beta$ (defined by $\tan \beta=v_{1} / v_{2}$ ) but rather by two different angles. Finally, we derived the expressions for the Higgs-fermion-fermion couplings in the presence of dimension-six operators.

Even though a study of phenomenological applications of our framework is beyond the scope of this article, we pointed out one interesting example illustrating the differences between 2HDM-EFT and SM-EFT. In the latter, there is only one dimension-six operator giving rise to fermion-fermion-Higgs-Higgs interactions and its effect on pair production of SM Higgses is limited by constraints on Higgs-fermion-fermion couplings. In the 2HDM-EFT instead, there are three operators entering Higgs-fermion-fermion couplings, whose contributions can cancel each other. The same operators, as well as additional ones, also con- 
tribute to the couplings of Higgs pairs to fermions but with different prefactors. Higgs pair production is decoupled from single-Higgs-fermion interactions and could thus be sizable.

\section{Acknowledgments}

We thank Michael Spira for useful discussions and for proofreading the article. A.C. is supported by an Ambizione Fellowship of the Swiss National Science Foundation. M.P. is supported by a Marie Curie Intra-European Fellowship of the European Community's 7th Framework Programme under contract number PIEF-GA-2013-622527.

Open Access. This article is distributed under the terms of the Creative Commons Attribution License (CC-BY 4.0), which permits any use, distribution and reproduction in any medium, provided the original author(s) and source are credited.

\section{References}

[1] T.D. Lee, A theory of spontaneous T violation, Phys. Rev. D 8 (1973) 1226 [INSPIRE].

[2] J.F. Gunion, H.E. Haber, G.L. Kane and S. Dawson, The Higgs hunter's guide, Front. Phys. 80 (2000) 1 [INSPIRE].

[3] G.C. Branco, P.M. Ferreira, L. Lavoura, M.N. Rebelo, M. Sher and J.P. Silva, Theory and phenomenology of two-Higgs-doublet models, Phys. Rept. 516 (2012) 1 [arXiv:1106.0034] [INSPIRE].

[4] J.E. Kim, Light pseudoscalars, particle physics and cosmology, Phys. Rept. 150 (1987) 1 [INSPIRE].

[5] R.D. Peccei and H.R. Quinn, CP conservation in the presence of instantons, Phys. Rev. Lett. 38 (1977) 1440 [INSPIRE].

[6] M. Trodden, Electroweak baryogenesis, Rev. Mod. Phys. 71 (1999) 1463 [hep-ph/9803479] [INSPIRE].

[7] A. Crivellin, C. Greub and A. Kokulu, Explaining $B \rightarrow D \tau \nu, B \rightarrow D^{*} \tau \nu$ and $B \rightarrow \tau \nu$ in a 2 HDM of type-III, Phys. Rev. D 86 (2012) 054014 [arXiv:1206.2634] [INSPIRE].

[8] A. Celis, M. Jung, X.-Q. Li and A. Pich, Sensitivity to charged scalars in $B \rightarrow D^{(*)} \tau \nu_{\tau}$ and $B \rightarrow \tau \nu_{\tau}$ decays, JHEP 01 (2013) 054 [arXiv:1210.8443] [INSPIRE].

[9] A. Crivellin, J. Heeck and P. Stoffer, A perturbed lepton-specific two-Higgs-doublet model facing experimental hints for physics beyond the Standard Model, Phys. Rev. Lett. 116 (2016) 081801 [arXiv:1507.07567] [INSPIRE].

[10] P. Fayet and S. Ferrara, Supersymmetry, Phys. Rept. 32 (1977) 249 [inSPIRE].

[11] P. Fayet, Supersymmetry and weak, electromagnetic and strong interactions, Phys. Lett. B 64 (1976) 159 [INSPIRE].

[12] H.E. Haber and G.L. Kane, The search for supersymmetry: probing physics beyond the Standard Model, Phys. Rept. 117 (1985) 75 [INSPIRE].

[13] M. Carena, S. Heinemeyer, O. Stål, C.E.M. Wagner and G. Weiglein, MSSM Higgs boson searches at the LHC: benchmark scenarios after the discovery of a Higgs-like particle, Eur. Phys. J. C 73 (2013) 2552 [arXiv:1302.7033] [INSPIRE]. 
[14] Particle Data Group collaboration, J. Beringer et al., Review of particle physics (RPP), Phys. Rev. D 86 (2012) 010001 [InSPIRE].

[15] M. Misiak et al., Updated NNLO QCD predictions for the weak radiative B-meson decays, Phys. Rev. Lett. 114 (2015) 221801 [arXiv:1503.01789] [INSPIRE].

[16] A. Crivellin, G. D'Ambrosio and J. Heeck, Explaining $h \rightarrow \mu^{ \pm} \tau^{\mp}, B \rightarrow K^{*} \mu^{+} \mu^{-}$and $B \rightarrow K \mu^{+} \mu^{-} / B \rightarrow K e^{+} e^{-}$in a two-Higgs-doublet model with gauged $L_{\mu}-L_{\tau}$, Phys. Rev. Lett. 114 (2015) 151801 [arXiv:1501.00993] [INSPIRE].

[17] A. Crivellin, G. D'Ambrosio and J. Heeck, Addressing the LHC flavor anomalies with horizontal gauge symmetries, Phys. Rev. D 91 (2015) 075006 [arXiv:1503.03477] [InSPIRE].

[18] S. Weinberg, Baryon and lepton nonconserving processes, Phys. Rev. Lett. 43 (1979) 1566 [INSPIRE].

[19] W. Buchmüller and D. Wyler, Effective Lagrangian analysis of new interactions and flavor conservation, Nucl. Phys. B 268 (1986) 621 [INSPIRE].

[20] C.J.C. Burges and H.J. Schnitzer, Virtual effects of excited quarks as probes of a possible new hadronic mass scale, Nucl. Phys. B 228 (1983) 464 [INSPIRE].

[21] C.N. Leung, S.T. Love and S. Rao, Low-energy manifestations of a new interaction scale: operator analysis, Z. Phys. C 31 (1986) 433 [INSPIRE].

[22] K. Hagiwara, R. Szalapski and D. Zeppenfeld, Anomalous Higgs boson production and decay, Phys. Lett. B 318 (1993) 155 [hep-ph/9308347] [INSPIRE].

[23] B. Grzadkowski, M. Iskrzynski, M. Misiak and J. Rosiek, Dimension-six terms in the Standard Model Lagrangian, JHEP 10 (2010) 085 [arXiv:1008.4884] [INSPIRE].

[24] C. Degrande et al., Effective field theory: a modern approach to anomalous couplings, Annals Phys. 335 (2013) 21 [arXiv:1205.4231] [INSPIRE].

[25] M. Trott, On the consistent use of constructed observables, JHEP 02 (2015) 046 [arXiv: 1409.7605] [INSPIRE].

[26] E. Masso, An effective guide to beyond the Standard Model physics, JHEP 10 (2014) 128 [arXiv:1406.6376] [INSPIRE].

[27] B. Henning, X. Lu and H. Murayama, How to use the Standard Model effective field theory, JHEP 01 (2016) 023 [arXiv:1412.1837] [INSPIRE].

[28] R. Contino, A. Falkowski, F. Goertz, C. Grojean and F. Riva, On the validity of the effective field theory approach to SM precision tests, JHEP 07 (2016) 144 [arXiv:1604.06444] [INSPIRE].

[29] G.F. Giudice, C. Grojean, A. Pomarol and R. Rattazzi, The strongly-interacting light Higgs, JHEP 06 (2007) 045 [hep-ph/0703164] [INSPIRE].

[30] R. Contino, M. Ghezzi, C. Grojean, M. Mühlleitner and M. Spira, Effective Lagrangian for a light Higgs-like scalar, JHEP 07 (2013) 035 [arXiv:1303.3876] [INSPIRE].

[31] A. Alloul, B. Fuks and V. Sanz, Phenomenology of the Higgs effective Lagrangian via FEYNRULES, JHEP 04 (2014) 110 [arXiv: 1310.5150] [INSPIRE].

[32] R. Contino, M. Ghezzi, C. Grojean, M. Mühlleitner and M. Spira, eHDECAY: an implementation of the Higgs effective Lagrangian into HDECAY,

Comput. Phys. Commun. 185 (2014) 3412 [arXiv:1403.3381] [INSPIRE]. 
[33] C.-Y. Chen, S. Dawson and C. Zhang, Electroweak effective operators and Higgs physics, Phys. Rev. D 89 (2014) 015016 [arXiv:1311.3107] [InSPIRE].

[34] C. Englert et al., Precision measurements of Higgs couplings: implications for new physics scales, J. Phys. G 41 (2014) 113001 [arXiv:1403.7191] [InSPIRE].

[35] A. Biekötter, A. Knochel, M. Krämer, D. Liu and F. Riva, Vices and virtues of Higgs effective field theories at large energy, Phys. Rev. D 91 (2015) 055029 [arXiv:1406.7320] [InSPIRE].

[36] F. Bonnet, M.B. Gavela, T. Ota and W. Winter, Anomalous Higgs couplings at the LHC and their theoretical interpretation, Phys. Rev. D 85 (2012) 035016 [arXiv:1105.5140] [INSPIRE].

[37] F. Bonnet, T. Ota, M. Rauch and W. Winter, Interpretation of precision tests in the Higgs sector in terms of physics beyond the Standard Model, Phys. Rev. D 86 (2012) 093014 [arXiv: 1207.4599] [INSPIRE].

[38] A. Crivellin, S. Najjari and J. Rosiek, Lepton flavor violation in the Standard Model with general dimension-six operators, JHEP 04 (2014) 167 [arXiv: 1312.0634] [INSPIRE].

[39] A. Crivellin, M. Hoferichter and M. Procura, Improved predictions for $\mu \rightarrow e$ conversion in nuclei and Higgs-induced lepton flavor violation, Phys. Rev. D 89 (2014) 093024 [arXiv: 1404.7134] [INSPIRE].

[40] A. Crivellin and S. Pokorski, Can the differences in the determinations of $V_{u b}$ and $V_{c b}$ be explained by new physics?, Phys. Rev. Lett. 114 (2015) 011802 [arXiv:1407.1320] [InSPIRE].

[41] G.M. Pruna and A. Signer, The $\mu \rightarrow$ e $\gamma$ decay in a systematic effective field theory approach with dimension 6 operators, JHEP 10 (2014) 014 [arXiv:1408.3565] [INSPIRE].

[42] B. Bhattacharya, A. Datta, D. London and S. Shivashankara, Simultaneous explanation of the $R_{K}$ and $R\left(D^{(*)}\right)$ puzzles, Phys. Lett. B 742 (2015) 370 [arXiv:1412.7164] [INSPIRE].

[43] R. Alonso, B. Grinstein and J. Martin Camalich, $\mathrm{SU}(2) \times \mathrm{U}(1)$ gauge invariance and the shape of new physics in rare B decays, Phys. Rev. Lett. 113 (2014) 241802 [arXiv: 1407.7044] [INSPIRE].

[44] A.J. Buras, J. Girrbach-Noe, C. Niehoff and D.M. Straub, $B \rightarrow K^{(*)} \nu \bar{\nu}$ decays in the Standard Model and beyond, JHEP 02 (2015) 184 [arXiv: 1409.4557] [INSPIRE].

[45] R. Alonso, B. Grinstein and J. Martin Camalich, Lepton universality violation and lepton flavor conservation in B-meson decays, JHEP 10 (2015) 184 [arXiv: 1505. 05164] [INSPIRE].

[46] L. Calibbi, A. Crivellin and T. Ota, Effective field theory approach to $b \rightarrow \operatorname{s\ell \ell }^{(\prime)}, B \rightarrow K^{(*)} \nu \bar{\nu}$ and $B \rightarrow D^{(*)} \tau \nu$ with third generation couplings, Phys. Rev. Lett. 115 (2015) 181801 [arXiv: 1506. 02661] [INSPIRE].

[47] J. Aebischer, A. Crivellin, M. Fael and C. Greub, Matching of gauge invariant dimension-six operators for $b \rightarrow s$ and $b \rightarrow c$ transitions, JHEP 05 (2016) 037 [arXiv:1512.02830] [INSPIRE].

[48] L. Berthier and M. Trott, Towards consistent electroweak precision data constraints in the SMEFT, JHEP 05 (2015) 024 [arXiv: 1502 .02570] [inSPIRE].

[49] J. Ellis, V. Sanz and T. You, Complete Higgs sector constraints on dimension-6 operators, JHEP 07 (2014) 036 [arXiv: 1404.3667] [INSPIRE].

[50] A. Falkowski and F. Riva, Model-independent precision constraints on dimension-6 operators, JHEP 02 (2015) 039 [arXiv: 1411.0669] [InSPIRE]. 
[51] I. Low, R. Rattazzi and A. Vichi, Theoretical constraints on the Higgs effective couplings, JHEP 04 (2010) 126 [arXiv:0907.5413] [INSPIRE].

[52] A. Pomarol and F. Riva, Towards the ultimate SM fit to close in on Higgs physics, JHEP 01 (2014) 151 [arXiv: 1308.2803] [INSPIRE].

[53] L. Berthier and M. Trott, Consistent constraints on the Standard Model effective field theory, JHEP 02 (2016) 069 [arXiv: 1508.05060] [INSPIRE].

[54] C. Englert, R. Kogler, H. Schulz and M. Spannowsky, Higgs coupling measurements at the LHC, Eur. Phys. J. C 76 (2016) 393 [arXiv:1511.05170] [inSPIRE].

[55] V. Cirigliano, W. Dekens, J. de Vries and E. Mereghetti, Constraining the top-Higgs sector of the Standard Model effective field theory, Phys. Rev. D 94 (2016) 034031 [arXiv: 1605.04311] [INSPIRE].

[56] C. Grojean, E.E. Jenkins, A.V. Manohar and M. Trott, Renormalization group scaling of Higgs operators and $\Gamma(h \rightarrow \gamma \gamma)$, JHEP 04 (2013) 016 [arXiv:1301.2588] [INSPIRE].

[57] E.E. Jenkins, A.V. Manohar and M. Trott, Renormalization group evolution of the Standard Model dimension six operators I: formalism and $\lambda$ dependence, JHEP 10 (2013) 087 [arXiv:1308.2627] [INSPIRE].

[58] E.E. Jenkins, A.V. Manohar and M. Trott, Renormalization group evolution of the Standard Model dimension six operators II: Yukawa dependence, JHEP 01 (2014) 035 [arXiv: 1310.4838] [INSPIRE].

[59] R. Alonso, E.E. Jenkins, A.V. Manohar and M. Trott, Renormalization group evolution of the Standard Model dimension six operators III: gauge coupling dependence and phenomenology, JHEP 04 (2014) 159 [arXiv:1312.2014] [INSPIRE].

[60] R. Gröber, M. Mühlleitner, M. Spira and J. Streicher, NLO QCD corrections to Higgs pair production including dimension-6 operators, JHEP 09 (2015) 092 [arXiv: 1504.06577] [INSPIRE].

[61] J. Elias-Miro, J.R. Espinosa, E. Masso and A. Pomarol, Higgs windows to new physics through $D=6$ operators: constraints and one-loop anomalous dimensions, JHEP 11 (2013) 066 [arXiv:1308.1879] [INSPIRE].

[62] J. Elias-Miró, J.R. Espinosa, E. Masso and A. Pomarol, Renormalization of dimension-six operators relevant for the Higgs decays $h \rightarrow \gamma \gamma, \gamma Z$, JHEP 08 (2013) 033 [arXiv:1302.5661] [INSPIRE].

[63] G. Passarino, NLO inspired effective Lagrangians for Higgs physics, Nucl. Phys. B 868 (2013) 416 [arXiv:1209.5538] [INSPIRE].

[64] M. Ghezzi, R. Gomez-Ambrosio, G. Passarino and S. Uccirati, NLO Higgs effective field theory and $\kappa$-framework, JHEP 07 (2015) 175 [arXiv:1505.03706] [INSPIRE].

[65] C. Hartmann and M. Trott, On one-loop corrections in the Standard Model effective field theory; the $\Gamma(h \rightarrow \gamma \gamma)$ case, JHEP 07 (2015) 151 [arXiv: 1505.02646] [INSPIRE].

[66] C. Hartmann and M. Trott, Higgs decay to two photons at one loop in the Standard Model effective field theory, Phys. Rev. Lett. 115 (2015) 191801 [arXiv:1507.03568] [INSPIRE].

[67] R. Gauld, B.D. Pecjak and D.J. Scott, One-loop corrections to $h \rightarrow b \bar{b}$ and $h \rightarrow \tau \bar{\tau}$ decays in the Standard Model dimension-6 EFT: four-fermion operators and the large-m $m_{t}$ limit, JHEP 05 (2016) 080 [arXiv: 1512.02508] [INSPIRE]. 
[68] ATLAS collaboration, Search for resonances in diphoton events at $\sqrt{s}=13 \mathrm{TeV}$ with the ATLAS detector, JHEP 09 (2016) 001 [arXiv: 1606.03833] [INSPIRE].

[69] CMS collaboration, Search for resonant production of high-mass photon pairs in proton-proton collisions at $\sqrt{s}=8$ and 13 TeV, Phys. Rev. Lett. 117 (2016) 051802 [arXiv: 1606.04093] [INSPIRE].

[70] W. Altmannshofer, J. Galloway, S. Gori, A.L. Kagan, A. Martin and J. Zupan, $750 \mathrm{GeV}$ diphoton excess, Phys. Rev. D 93 (2016) 095015 [arXiv: 1512.07616] [INSPIRE].

[71] R.S. Gupta, S. Jäger, Y. Kats, G. Perez and E. Stamou, Interpreting a $750 \mathrm{GeV}$ diphoton resonance, JHEP 07 (2016) 145 [arXiv: 1512.05332] [INSPIRE].

[72] N. Bizot, S. Davidson, M. Frigerio and J.L. Kneur, Two Higgs doublets to explain the excesses $p p \rightarrow \gamma \gamma(750 \mathrm{GeV})$ and $h \rightarrow \tau^{ \pm} \mu^{\mp}, J H E P 03$ (2016) 073 [arXiv : 1512.08508] [INSPIRE].

[73] X.-F. Han, L. Wang and J.M. Yang, An extension of two-Higgs-doublet model and the excesses of $750 \mathrm{GeV}$ diphoton, muon g-2 and $h \rightarrow \mu \tau$, Phys. Lett. B 757 (2016) 537 [arXiv: 1601.04954] [INSPIRE].

[74] A. Angelescu, A. Djouadi and G. Moreau, Scenarii for interpretations of the LHC diphoton excess: two Higgs doublets and vector-like quarks and leptons, Phys. Lett. B 756 (2016) 126 [arXiv: 1512.04921] [INSPIRE].

[75] S. Di Chiara, L. Marzola and M. Raidal, First interpretation of the $750 \mathrm{GeV}$ diphoton resonance at the LHC, Phys. Rev. D 93 (2016) 095018 [arXiv:1512.04939] [INSPIRE].

[76] M. Low, A. Tesi and L.-T. Wang, A pseudoscalar decaying to photon pairs in the early LHC Run 2 data, JHEP 03 (2016) 108 [arXiv:1512.05328] [INSPIRE].

[77] S. Moretti and K. Yagyu, $750 \mathrm{GeV}$ diphoton excess and its explanation in two-Higgs-doublet models with a real inert scalar multiplet, Phys. Rev. D 93 (2016) 055043 [arXiv: 1512.07462] [INSPIRE].

[78] E. Bertuzzo, P.A.N. Machado and M. Taoso, Di-photon excess in the 2HDM: hasting towards the instability and the non-perturbative regime, arXiv:1601.07508 [INSPIRE].

[79] A.E. Cárcamo Hernández, I. de Medeiros Varzielas and E. Schumacher, The $750 \mathrm{GeV}$ diphoton resonance in the light of a $2 H D M$ with $S_{3}$ flavour symmetry, arXiv:1601.00661 [INSPIRE].

[80] M.A. Perez, J.J. Toscano and J. Wudka, Two photon processes and effective Lagrangians with an extended scalar sector, Phys. Rev. D 52 (1995) 494 [hep-ph/9506457] [INSPIRE].

[81] J.F. Gunion and H.E. Haber, The CP conserving two Higgs doublet model: the approach to the decoupling limit, Phys. Rev. D 67 (2003) 075019 [hep-ph/0207010] [INSPIRE].

[82] J.D. Bjorken and S. Weinberg, A mechanism for nonconservation of muon number, Phys. Rev. Lett. 38 (1977) 622 [INSPIRE].

[83] B. McWilliams and L.-F. Li, Virtual effects of Higgs particles, Nucl. Phys. B 179 (1981) 62 [INSPIRE].

[84] T.P. Cheng and M. Sher, Mass matrix ansatz and flavor nonconservation in models with multiple Higgs doublets, Phys. Rev. D 35 (1987) 3484 [INSPIRE].

[85] A. Crivellin, A. Kokulu and C. Greub, Flavor-phenomenology of two-Higgs-doublet models with generic Yukawa structure, Phys. Rev. D 87 (2013) 094031 [arXiv:1303.5877] [INSPIRE]. 
[86] S. Dawson, S. Dittmaier and M. Spira, Neutral Higgs boson pair production at hadron colliders: QCD corrections, Phys. Rev. D 58 (1998) 115012 [hep-ph/9805244] [INSPIRE].

[87] A. Djouadi, W. Kilian, M. Mühlleitner and P.M. Zerwas, Production of neutral Higgs boson pairs at LHC, Eur. Phys. J. C 10 (1999) 45 [hep-ph/9904287] [INSPIRE].

[88] J. Baglio, A. Djouadi, R. Gröber, M. Mühlleitner, J. Quevillon and M. Spira, The measurement of the Higgs self-coupling at the LHC: theoretical status, JHEP 04 (2013) 151 [arXiv: 1212.5581] [INSPIRE].

[89] R. Frederix et al., Higgs pair production at the LHC with NLO and parton-shower effects, Phys. Lett. B 732 (2014) 142 [arXiv:1401.7340] [INSPIRE].

[90] F. Goertz, A. Papaefstathiou, L.L. Yang and J. Zurita, Higgs boson pair production in the $D=6$ extension of the SM, JHEP 04 (2015) 167 [arXiv:1410.3471] [INSPIRE]. 